Supporting Information

\title{
Immobilized Multifunctional Polymersomes on Solid Surfaces: Infrared Light-Induced Selective Photochemical Reactions, pH Responsive Behavior and Probing Mechanical Properties under Liquid Phase
}

Banu Iyisan ${ }^{\dagger, \ldots,}$, Andreas Janke ${ }^{\dagger}$, Philipp Reichenbach ${ }^{\|}$, Lukas M. Eng ${ }^{\| l, \#}$, Dietmar Appelhans ${ }^{*}, \dagger$,

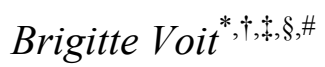

${ }^{\dagger}$ Leibniz Institut für Polymerforschung Dresden e.V. Hohe Strasse 6, 01069 Dresden, Germany

Organic Chemistry of Polymers, Technische Universität Dresden, 01062 Dresden, Germany

${ }^{\S}$ International Helmholtz Research School for Nanoelectronic Networks, 01328 Dresden,

Germany

"Institut für Angewandte Photophysik, Technische Universität Dresden, 01062 Dresden, Germany

\#Center for Advancing Electronic Devices Dresden, Technische Universität Dresden, Würzburger Straße 46, 01187 Dresden, Germany

*E-mail: voit@ipfdd.de; applhans@ipfdd.de 
Table of Contents for Supporting Information:

Page

1. Formation and Cross-linking of Polymersomes 3

2. NVOC Modification of Polymersomes 3

3. Cryogenic Transmission Electron Microscopy (cryo-TEM) Protocol 4

4. Supporting Figures $\quad 5$

Figure S1. cryo-TEM micrographs of PS1 and PS1C polymersomes at basic and 5 acidic condition

Figure S2. (a) Peak force tapping mode liquid AFM height image of PS0-S3

polymersomes without adamantane groups at $\mathrm{pH} 7.4$ condition (b) Height vs diameter relationship for PS0-S3 polymersomes.

5. References 


\section{Formation and Cross-linking of Polymersomes}

Polymersomes were prepared as described previously. ${ }^{1}$ Briefly, a mixture of block copolymers was dissolved in aqueous $\mathrm{HCl}$ solution $(\mathrm{pH}$ 2) then passed through $0.2 \mu \mathrm{m}$ nylon filter to remove any impurities. To initiate the self-assembly process, $\mathrm{pH}$ was slowly increased to $\mathrm{pH} 9$ by adding $1 \mathrm{M} \mathrm{NaOH}$. Finally, polymersomes were formed after four days of stirring in dark condition. To perform photo cross-linking of the polymersomes, the solution was subsequently passed through the $0.8 \mu \mathrm{m}$ nylon filter and then was placed into the UV chamber equipped with an iron lamp, 80 $\mathrm{mW} / \mathrm{cm}^{2}$, (UVACUBE100, honle UV Technologies, Germany) for irradiation of 30 minutes.

\section{NVOC Modification of Polymersomes}

- Synthesis of Nitroveratryloxycarbonyl (NVOC) Protected Amine Groups<smiles>C#CCNC(=O)C(C#CCNC(=O)OCc1cc(OC)c(OC)cc1[N+](=O)[O-])C(=O)O</smiles>

To an aqueous mixture of propargylamine $(0.11 \mathrm{~mL}, 1.7 \mathrm{mmol})$ and sodium bicarbonate $(0.3 \mathrm{~g}$, $3.6 \mathrm{mmol})$, a solution of nitroveratryloxycarbonylchloride $(0.5 \mathrm{~g}, 1.8 \mathrm{mmol})$ in THF was added dropwise. After 15 hours of stirring at room temperature, the precipitation was filtered and THF was evaporated under reduced pressure. The obtained suspension was diluted with water and extracted with ethyl acetate followed by drying with sodium sulfate. Then ethyl acetate was evaporated to obtain the solid product (yield 88\%). Since the product was not pure enough determined by NMR spectra, it was further purified with column chromatography on silica gel using $n$-hexane/ethyl acetate $=1: 2$ (Yield: $50 \%){ }^{1}$ 
${ }^{1} \mathbf{H}$ NMR (500.13 MHz, $\left.\mathbf{C D C l}_{3}, \boldsymbol{\delta}\right): 2.26$ (t, J=2.5 Hz, $1 \mathrm{H}$ ), 3.96 (s, $\left.3 \mathrm{H}\right), 3.99$ (s, $\left.3 \mathrm{H}\right), 4.03$ (dd, $\mathrm{J}=5.7,2.5 \mathrm{~Hz}, 2 \mathrm{H}), 5.07(\mathrm{br}, \mathrm{NH}), 5.55(\mathrm{~s}, 2 \mathrm{H}), 7.0(\mathrm{~s}, 1 \mathrm{H}), 7.72(\mathrm{~s}, 1 \mathrm{H})$.

${ }^{13}$ C NMR (125.76 MHz, $\left.\mathbf{C D C l}_{3}, \boldsymbol{\delta}\right): 31.00$ (C-d), 56.41 (C-b, C-c), 63.85 (C-f), 71.77 (C-a), 79.43 (C), 108.23 (C-g), 110.31 (C-h), 127.67 (C- i), 139.92 (C-m), 148.22 (C-l), 153.56 (C-k), $155.37(\mathrm{C}-\mathrm{j})$.

\section{- NVOC Conjugation to the Surface of the Polymersomes}

NVOC modification of polymersomes for photo chemical reactions was performed as described in our recent publication. ${ }^{1}$ The aqueous solutions of $\mathrm{CuSO}_{4} .5 \mathrm{H}_{2} \mathrm{O}(0.25$ mol eq. $)$, sodium ascorbate (0.5 mol eq.), TBTA ( 0.25 mol eq. in DMSO), and alkyne modified NVOC groups (1.5 mol eq. in DMSO) were added to the $1 \mathrm{mg} / \mathrm{mL}$ polymersome solution at $\mathrm{pH} 8$ (PS1 or PS2, azide groups, $1 \mathrm{~mol}$ eq.). The reaction mixture was stirred for 2 days at room temperature. Subsequently, the polymersome solution was transferred to a dialysis membrane (5 kDa MWCO) and extensively dialyzed against EDTA solution in millipore water $(0.055 \mathrm{mM}, \mathrm{pH} 8)$ for 2 days.

\section{Cryogenic Transmission Electron Microscopy (cryo-TEM) Protocol}

Cryo-TEM images were obtained using Libra 120 microscope (Carl Zeiss Microscopy GmbH, Oberkochen, Germany) at an acceleration voltage of $120 \mathrm{kV}$. Samples were prepared by dropping $1 \mu \mathrm{l}$ of polymersome solution $(1 \mathrm{mg} / \mathrm{mL})$ on each side of a copper grid coated with holey carbon foil (so-called Lacey type). A piece of filter paper was used to remove the excess water; the sample was then rapidly frozen in liquid ethane at $-178^{\circ} \mathrm{C}$. The blotting with the filter paper and plunging into liquid ethane was done in a Leica GP device (Leica Microsystems GmbH, Wetzlar, Germany). All images were recorded in bright field at $-172{ }^{\circ} \mathrm{C}$. 


\section{Supporting Figures}
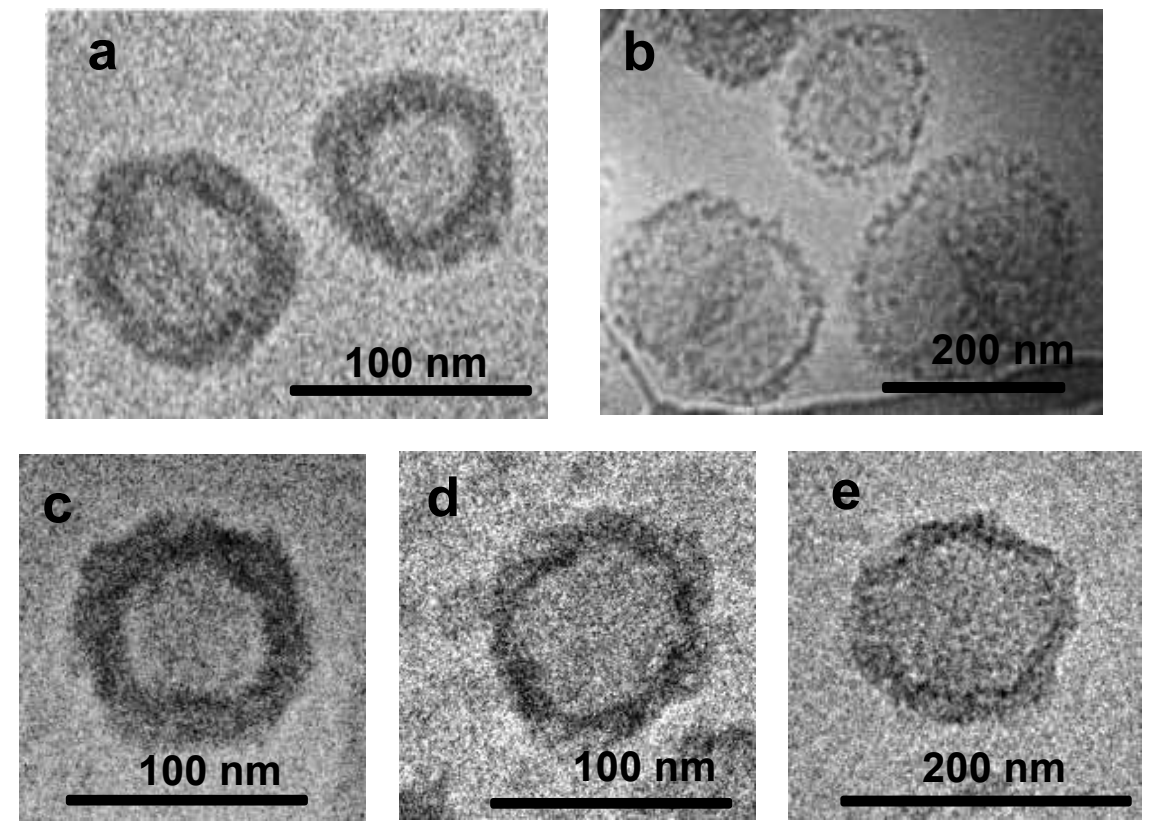

Figure S1. cryo-TEM micrographs of PS1 polymersomes at pH 9 (a,c), pH 5 (b) condition. cryo-TEM micrographs of UV irradiated PS1C polymersomes at $\mathrm{pH} 8$ (d), $\mathrm{pH} 5$ (e) condition.
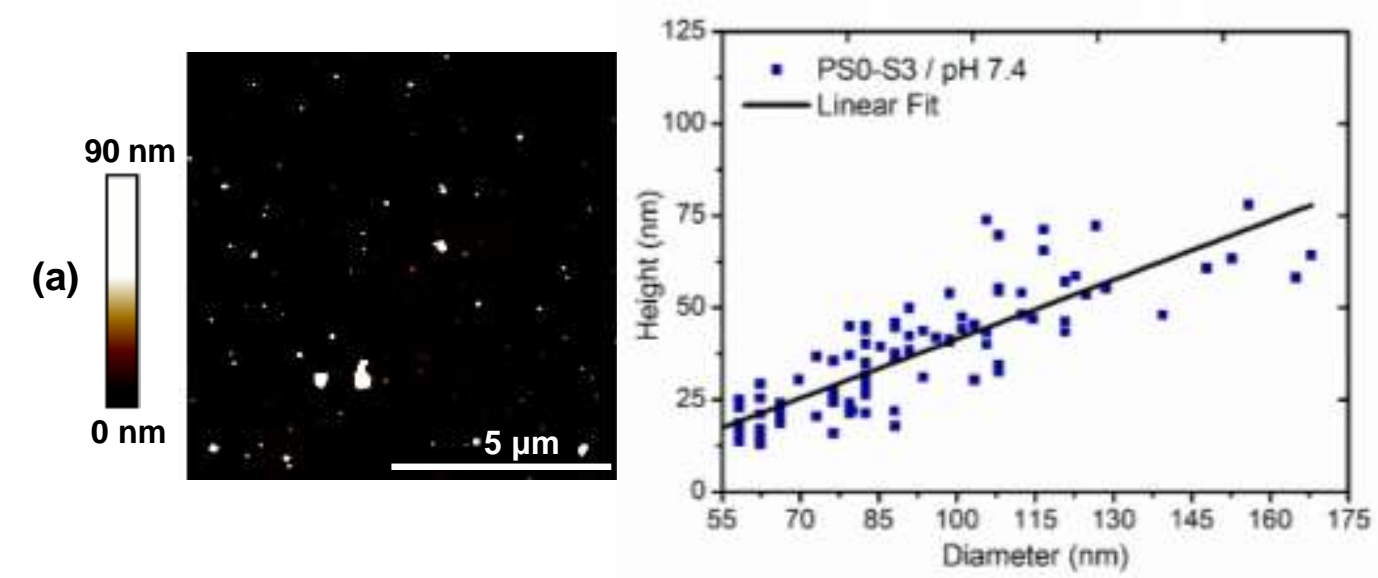

(b)

Figure S2. (a) Peak force tapping mode liquid AFM height image of PS0-S3 polymersomes without adamantane groups at $\mathrm{pH} 7.4$ condition (b) Height vs diameter relationship for PS0-S3 polymersomes. (PS0: Diameter $=117 \pm 1.1 \mathrm{~nm}$ and $\mathrm{PDI}=0.19 \pm 0.03$ obtained from DLS analysis self assembled from $\mathrm{BC} 2$ polymer and contains no functional groups) 


\section{References}

(1) Iyisan, B.; Kluge, J.; Formanek, P.; Voit, B.; Appelhans, D., Multifunctional and DualResponsive Polymersomes as Robust Nanocontainers: Design, Formation by Sequential Post-

Conjugations, and pH-Controlled Drug Release. Chem. Mater. 2016, DOI: 10.1021/acs.chemmater.5b05016 